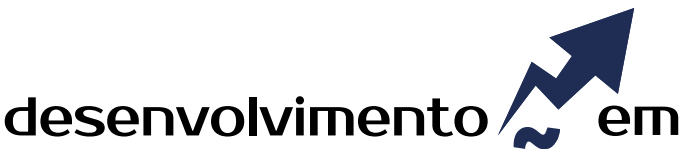 QUESTÃO
}

\section{Desenvolvimento Humano dos Municípios de Mato Grosso e Mato Grosso do Sul Contidos no Bioma Pantanal}

\author{
http://dx.doi.org/10.21527/2237-6453.2018.45.82-96
}

Recebido em: 26/4/2017

Aceito em: 26/6/2018

\author{
Junior Miranda Scheuer ${ }^{1}$, Sandra Mara Alves da Silva Neves ${ }^{2}$, \\ Edinéia Aparecida dos Santos Galvanin ${ }^{3}$, Ronaldo José Neves (In memoriam) ${ }^{4}$
}

\begin{abstract}
RESUMO
Objetivou-se avaliar, por meio do Índice de Desenvolvimento Humano (IDHM) dos municípios do bioma Pantanal, o desenvolvimento humano da fundação e/ou emancipação municipal, estabelecendo comparações ao IDHM dos municípios pantaneiros com o IDH brasileiro e dos Estados de Mato Grosso (MT) e Mato Grosso do Sul (MS). Selecionou-se os municípios de MT e MS contidos no bioma, agrupados por data de fundação/emancipação em períodos de 50 anos. Os municípios fundados no século 18 apresentaram os melhores resultados no IDHM $(\mathrm{m})$ e nos indicadores de renda $(\mathrm{m})$ e educação $(\mathrm{m})$. Os emancipados em 1913 a 1963 destacaram-se no de longevidade $(\mathrm{m})$. Os emancipados entre 1811 a 1861 perfizeram os menores valores para o IDHM $(m)$, renda $(m)$, educação $(m)$ e longevidade $(m)$. Os municípios de MT e MS contidos no bioma exibiram resultados inferiores quando comparado aos Estados e ao Brasil. A emancipação que não observa a sustentabilidade socioestrutural pode resultar na criação de municípios sem as características básicas de manutenção e de suporte ao desenvolvimento humano, a exemplo de Barão de Melgaço e Bodoquena. Concluiu-se que o desenvolvimento humano da fundação/emancipação dos municípios não se associou, diretamente, com os períodos de sua criação, ou seja, os mais antigos, de maneira geral, não se sobressaíram aos mais novos, e vice-versa. Ademais, questões como o perfil da gestão e administração pública, políticas públicas, localização geográfica, riquezas naturais, infraestrutura etc., podem contribuir significativamente com os resultados do IDHM tanto positiva quanto negativamente.
\end{abstract}

Palavras-chave: Renda. Longevidade. Educação. Emancipação. Geotecnologias.

HUMAN DEVELOPMENT OF MUNICIPALITIES OF MATO GROSSO AND MATO GROSSO DO SUL CONTAINED IN BIOMA PANTANAL

\begin{abstract}
Aimed to evaluate, through the Human Development Index (IDHM) in the municipalities of the Pantanal biome, the human development of the foundation/emancipation municipal, establishing comparisons to the IDHM of the Pantanal municipalities with the Brazilian IDH and the states of Mato Grosso (MT) and Mato Grosso do Sul (MS). The municipalities of MT and MS were selected from the biome, grouped by date of foundation/emancipation in periods of 50 years. The municipalities founded in the 18th century showed the best results in the IDHM $(\mathrm{m})$ and indicators of income $(\mathrm{m})$ and education $(\mathrm{m})$. The emancipated ones in 1913 to 1963 stood out in longevity $(\mathrm{m})$. The emancipated between 1811 and 1861 had the lowest values for the IDHM $(m)$, income $(m)$, education $(m)$ and longevity $(m)$. The municipalities of MT and MS contained in the biome showed inferior results when compared to the states and to Brazil. The emancipation that does not observe socio-structural sustainability can result in the creation of municipalities without the basic characteristics of maintenance and support to human development, like Barão de Melgaço and Bodoquena. It was concluded that the human development of the foundation/ emancipation of the municipalities were not directly associated with the periods of its creation, that is, the oldest, in general, did not stand out to the younger ones, and vice versa. In addition, issues such as the profile of public management and administration, public policies, geographic location, natural wealth, infrastructure, etc. can contribute significantly to the results of the IDHM both positively and negatively.
\end{abstract}

Keywords: Income. Longevity. Education. Emancipation. Geotechnologies.

\footnotetext{
${ }^{1}$ Mestre em Ambiente e Sistemas de Produção Agrícola pela Universidade do Estado de Mato Grosso (Unemat), campus de Tangará da Serra/MT. Doutorando em Ciências Agrárias pela Universidad de la República (Udelar), campus de Montevidéu, Uruguai. Professor na Facultad de Agronomia da Universidad de la República (Udelar).juniorscheuer@yahoo.com.br

${ }^{2}$ Doutora em Geografia pela Universidade Federal do Rio de Janeiro (UFRJ). Professora dos Programas de Pós-Graduação em Ambiente e Sistemas de Produção e de Geografia da Universidade do Estado de Mato Grosso (Unemat). ssneves@unemat.br

${ }^{3}$ Doutora em Ciências Cartográficas pela Universidade Estadual Paulista Júlio de Mesquita Filho (Unesp). Professora do Programa de Pós-Graduação em Ambiente e Sistemas de Produção Agrícola da Universidade do Estado de Mato Grosso (Unemat). galvaninbbg@unemat.br

${ }^{4}$ Doutor em Geografia pela Universidade Federal do Rio de Janeiro (In memoriam). Era professor dos Programas de Pós-Graduação em Ambiente e Sistemas de Produção e de Geografia da Universidade do Estado de Mato Grosso (Unemat).
} 
O entendimento do conceito de desenvolvimento humano está mais "humanizado" em relação à ótica inicial de avaliar o progresso de um país apenas por condicionantes econômicos. No ano de 1960 as Nações Unidas definiram a década como a do desenvolvimento, simplesmente por estipular um crescimento econômico de $5 \%$ ao ano àqueles países subdesenvolvidos, o que não resultou em melhorias das condições de vida da população. Nos anos de 1970, então na segunda década do desenvolvimento, foram incluídas nas metas, além do crescimento econômico, a cooperação internacional, a geração de empregos e melhorias na educação e na saúde, visando, dessa manei$\mathrm{ra}$, a atender às necessidades humanas básicas, gerar empregos e, consequentemente, reduzir a pobreza avassaladora desse período (TORRES; FERREIRA; DINI, 2003; MACHADO; PAMPLONA, 2008).

Nessa ótica, discutir a perspectiva humana no conceito de desenvolvimento foi uma grande evolução (HAQ, 1995), e, na concepção de Batista Filho e Borba (2013, p. 1), o desenvolvimento humano envolve questões interdependentes, como a "economia, cultura, política, estruturas e funções sociais, território e seus recursos, ecologia, ética, coparticipação, subjetividade e a sustentabilidade como uma condição comum a todos os outros atributos".

Isto posto, os primeiros conceitos e instrumentos para aferir o desenvolvimento humano foram expressos no Relatório de Desenvolvimento Humano do Programa das Nações Unidas para o Desenvolvimento do ano de 1990 (UNITED..., 1990, p. 9), complementado no UNDP de 1992 (UNITED..., 1992, p. 17) e no UNDP de 2001 (UNITED..., 2001, p. 23), em que foi concebido enquanto:

(...) um processo de ampliação das escolhas das pessoas. Em princípio, estas escoIhas podem ser infinitas e mudar ao longo do tempo. Mas, em todos os níveis de desenvolvimento os três principais são levar uma vida longa e saudável para adquirir conhecimento e ter acesso aos recursos necessários para um padrão de vida decente. Se essas escolhas essenciais não estão disponíveis, muitas outras oportunidades permanecem inacessíveis (...). Se o desenvolvimento é ampliar o leque das escolhas das pessoas, deve fazê-lo, não só para as gerações atuais, mas para do futuro também. Ele deve ser sustentável (...). As capacidades básicas para o desenvolvimento humano são: ter uma vida longa e saudável, ser bem-informado, ter acesso aos recursos necessários para um padrão de vida decente e ser capaz de participar da vida da comunidade (Tradução nossa).

No Relatório de Desenvolvimento Humano do ano de 2014, revisou-se o conceito e estendeu-se a discussão sobre o desenvolvimento humano perpassando as questões da ampliação das escolhas e do acesso à educação, saúde, segurança e de uma vida razoável para um "grau de solidez dessas conquistas e da existência de condições suficientes para um desenvolvimento humano sustentado", ou seja, a inclusão no tema da vulnerabilidade e resiliência humana (UNITED..., 2014, p. 1).

Em sequência às concepções teóricas, Mahbub ul Haq (1995)e Amartya Sen (2010) criaram o Índice de Desenvolvimento Humano (IDH) para medir o desenvolvimento humano dos países. $O$ índice reúne os três componentes fundamentais para o desenvolvimento: padrão de vida (renda), longevidade (expectativa de vida ao nascer) e educação (conhecimento) (ATLAS BRASIL, 2013). 
Com vistas a adaptar o cálculo do IDH global às características dos municípios brasileiros, o Programa das Nações Unidas para o Desenvolvimento (PNUD Brasil), o Instituto de Pesquisa Econômica Aplicada (Ipea) e a Fundação João Pinheiro, desenvolveram a metodologia do IDH Municipal, usualmente chamado de IDHM, embasada nos três indicadores globais supracitados (ATLAS BRASIL, 2013).

A inciativa de verificar o IDHM viabilizou o conhecimento e a comparação da complexidade do desenvolvimento humano dos municípios, possibilitando o arranjo de informações, a espacialização dos índices e as tomadas de decisão por parte dos gestores públicos (federal, estadual e municipal), e, no caso deste estudo, a avaliação do desenvolvimento humano da fundação e/ou criação de municípios.

A fundação/criação de municípios a partir da emancipação dos distritos municipais, confere autonomia política (CIGOLINI, 2014), administrativa, financeira e normativa (PITTERI, 2005), contudo nem sempre há viabilidade econômica sustentável e condições básicas de desenvolvimento humano para a recaracterização do território.

Fleury (2003) e Veiga (2003) citaram que muitas vezes as motivações de criação dos municípios são mais por natureza política do que propriamente o desenvolvimento econômico de determinada localidade, resultando na dependência das transferências constitucionais do governo federal, em especial do Fundo de Participação dos Municípios (FPM), que acaba por constituir a principal fonte de receita dos novos e pequenos municípios (ABRUCIO; FRANZESE, 2007).

A criação de municípios, regulado pelo Estado, deve ou deveria garantir condições econômicas, sociais e culturais (ALBA, 2002; FERNANDES; ARAÚJO, 2015) por meio de políticas públicas, planos de desenvolvimento, Plano Diretor (VILLAÇA, 1999), etc., atuando não somente no sentido de gerenciá-los, mas também com vistas de proporcionar condições de autonomia e atender às necessidades da população (SAQUET, 2007), visando o estabelecimento estrutural local com eficiência econômica e qualidade de vida às pessoas (BUARQUE, 2006).

Diante do problema da emancipação de municípios sem a devida sustentabilidade socioestrutural, esta pesquisa investigou a fundação e/ou criação dos municípios dos Estados de Mato Grosso e Mato Grosso do Sul, contidos no bioma Pantanal, justificado por se tratar de uma das maiores extensões úmidas contínuas do planeta (BRASIL, 2017), fundamental para a conservação ambiental e a manutenção da biodiversidade brasileira (ANGEOLETTO et al., 2016).

Atualmente, a principal economia dos municípios inseridos no bioma Pantanal é a pecuária bovina de corte estabelecida em pastagem extensiva, ou seja, em campos abertos, ocasionando grande parte dos desmatamentos no bioma (ABDON et al., 2007), acrescidos dos impactos que recebe das atividades antrópicas realizadas nas áreas de planalto que o circundam e no próprio estabelecimento das cidades.

Destarte, o estudo objetivou avaliar, por meio do IDHM dos municípios do bioma Pantanal, o desenvolvimento humano da fundação e/ou emancipação municipal, estabelecendo comparações ao IDHM dos municípios pantaneiros com o IDH brasileiro e dos Estados de Mato Grosso e Mato Grosso do Sul. 
O presente artigo foi organizado em quatro partes, incluindo esta introdução do tema, que contextualiza o leitor sobre a evolução e abrangência do conceito de desenvolvimento humano e dos pontos básicos relativos à emancipação. Na parte dois apresenta-se a caracterização da área de estudo e os procedimentos metodológicos utilizados, no intuito de contemplar o objetivo proposto da pesquisa. Os resultados e as discussões da investigação constituíram a terceira parte, em que foram abordadas o desenvolvimento humano da fundação e/ou emancipação dos municípios inseridos no bioma Pantanal e a análise comparativa a partir do IDHM destes municípios pantaneiros com a dos Estados e do Brasil. A conclusão e as referências bibliográficas constituíram a última parte do artigo.

\section{MATERIAL E MÉTODOS}

\section{Área de estudo}

A área de pesquisa foi composta pelos municípios de Mato Grosso e Mato Grosso do Sul contidos no bioma Pantanal, que totalizam 227.447,17 km² (Figura 1) e 1.387.339 habitantes (INSTITUTO..., 2014).

A ocupação da região pantaneira iniciou no século 17 pelos bandeirantes paulistas com a captura dos índios para a comercialização nos mercados de escravos, a extração de minérios e por atividades agropecuárias, principalmente a cana-de-açúcar e a pecuária bovina. A partir dos anos de 0 do século 20, houve a migração de milhares de pessoas do Sul e do Sudeste do Brasil, incentivados por projetos do governo voltados ao desenvolvimento da agropecuária, a citar o Programa de Desenvolvimento dos Cerrados (Polocentro) e o Programa Integrado de Desenvolvimento do Noroeste do Brasil (Polonoroeste), ações que visavam a ocupar, produzir e integrar a região circundante ao Pantanal no cenário de desenvolvimento nacional (EMPRESA..., 2017).

Figura 1 - Distribuição dos municípios mato-grossenses e sul-mato-grossenses no bioma Pantanal

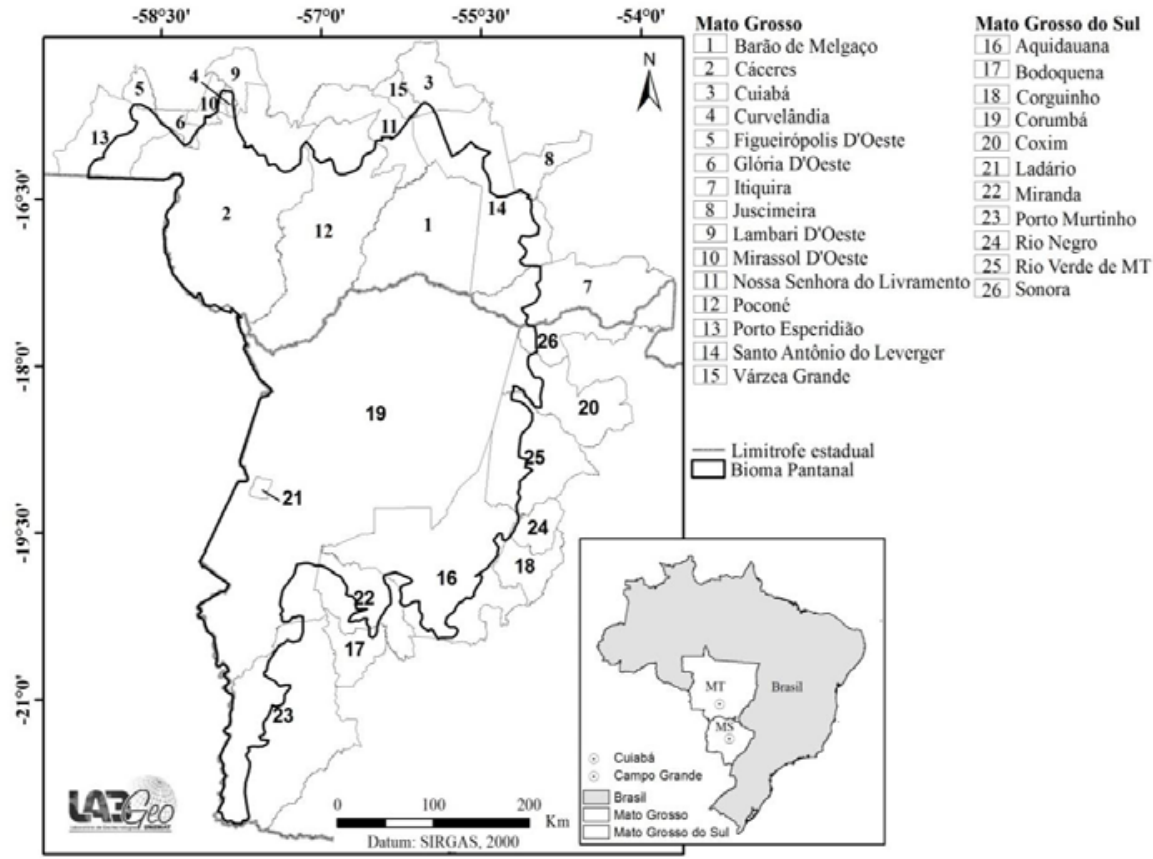

Fonte: UNIVERSIDADE..., 2017. 


\section{Procedimento metodológico}

Os municípios foram agrupados conforme a data de fundação e/ou emancipação em períodos de 50 anos, com exceção para o primeiro (século 18), que foi caracterizado por municípios fundados em um tempo superior à determinação inicial (Quadro 1).

Quadro 1 - Distribuição dos períodos de fundação e/ou criação dos municípios

\begin{tabular}{|c|c|c|c|}
\hline Período & Municípios & Estado & $\begin{array}{l}\text { Fundação e/ou } \\
\text { criação }\end{array}$ \\
\hline \multirow{3}{*}{$\begin{array}{l}\text { Primeiro período } \\
\text { - século } 18\end{array}$} & Cuiabá & \multirow{2}{*}{ Mato Grosso } & 1719 \\
\hline & Cáceres & & 1778 \\
\hline & Corumbá & Mato Grosso do Sul & 1778 \\
\hline \multirow{2}{*}{$\begin{array}{l}\text { Segundo período } \\
\text { - } 1811 \text { a } 1861\end{array}$} & Poconé & Mato Grosso & 1881 \\
\hline & Miranda & Mato Grosso do Sul & 1857 \\
\hline \multirow{4}{*}{$\begin{array}{l}\text { Terceiro período - } \\
\qquad 1862 \text { a } 1912\end{array}$} & Nossa Sra. do Livramento & Mato Grosso & 1883 \\
\hline & Coxim & Mato Grosso do Sul & 1898 \\
\hline & Santo Antônio do Leverger & Mato Grosso & 1890 \\
\hline & Porto Murtinho & Mato Grosso do Sul & 1911 \\
\hline \multirow{7}{*}{$\begin{array}{c}\text { Quarto período - } \\
1913 \text { a } 1963\end{array}$} & Aquidauana & Mato Grosso do Sul & 1918 \\
\hline & Várzea Grande & \multirow{3}{*}{ Mato Grosso } & 1948 \\
\hline & Barão de Melgaço & & \multirow{5}{*}{1953} \\
\hline & Itiquira & & \\
\hline & Corguinho & \multirow{3}{*}{ Mato Grosso do Sul } & \\
\hline & Ladário & & \\
\hline & Rio Verde de Mato Grosso & & \\
\hline \multirow{10}{*}{$\begin{array}{l}\text { Quinto período - } \\
1964 \text { a } 2014\end{array}$} & Rio Negro & Mato Grosso do Sul & 1964 \\
\hline & Mirassol D’Oeste & Mato Grosso & 1976 \\
\hline & Juscimeira & Mato Grosso & 1979 \\
\hline & Bodoquena & Mato Grosso do Sul & 1980 \\
\hline & Figueirópolis D’Oeste & \multirow{2}{*}{ Mato Grosso } & \multirow{2}{*}{1986} \\
\hline & Porto Esperidião & & \\
\hline & Sonora & Mato Grosso do Sul & 1988 \\
\hline & Lambari D’Oeste & \multirow{2}{*}{ Mato Grosso } & \multirow{2}{*}{1991} \\
\hline & Glória D’Oeste & & \\
\hline & Curvelândia & Mato Grosso & 1998 \\
\hline
\end{tabular}

Fonte: Adaptado da ASSOCIAÇÃO..., 2017; SECRETARIA..., 2017.

A análise foi verificada pelo IDHM e por seus indicadores: renda, longevidade e educação, de 2010 (ATLAS BRASIL, 2013). Entrementes, calculou-se o IDHM médio, renda média, longevidade média e educação média para os períodos determinados, representados pelas equações, a seguir descritas. 
onde:

- IDHM (m): Índice de Desenvolvimento Humano Municipal médio;

- $\sum$ r: somatória do indicador de renda do IDHM;

- $\Sigma$ I: somatória do indicador de longevidade do IDHM;

- ¿e: somatória do indicador de educação do IDHM.

$$
\mathrm{R}(\mathrm{m})=\frac{\sum r}{\mathrm{M}}
$$

onde:

$-\mathrm{R}(\mathrm{m})$ : Indicador de renda média;

- $\sum$ r: somatória do indicador de renda do IDHM;

- M: número de municípios no período de análise.

$$
\mathrm{L}(\mathrm{m})=\frac{\sum l}{\mathrm{M}}
$$

onde:

$-L(m)$ : Indicador de longevidade média;

- ¿l: somatória do indicador de longevidade do IDHM;

- M: número de municípios no período de análise.

$$
\mathrm{E}(\mathrm{m})=\frac{\sum e}{\mathrm{M}}
$$

onde:

- E (m): Indicador de educação média;

- ¿e: somatória do indicador de educação do IDHM;

- M: número de municípios no período de análise.

Os indicadores de renda, longevidade e educação quando desagregados do IDHM possibilitaram a análise e a compreensão da participação de cada um dos elementos que os compõem na construção do índice.

Na comparação do IDHM dos municípios pantaneiros com o IDHM nacional e estaduais utilizou-se a equação 5 :

$$
\operatorname{IDHM}(\mathrm{p})=\sqrt[3]{\sum r(m) \times \sum l(m) \times \sum e(m)}
$$

onde:

- IDHM (p): Índice de Desenvolvimento Humano dos municípios pantaneiros;

$-\sum r(m)$ : somatória do indicador de renda do IDHM nos períodos;

- $\Sigma(\mathrm{l})$ : somatória do indicador de longevidade do IDHM nos períodos;

$-\sum \mathrm{e}(\mathrm{m})$ : somatória do indicador de educação do IDHM nos períodos. 
Para os indicadores de renda, longevidade e educação fez-se uma média simples dos resultados encontrados nos cinco períodos de observação.

No estabelecimento da análise qualitativa do desenvolvimento do IDHM foram consideradas as seguintes classes e cores, conforme o Atlas Brasil (2013), para criação da legenda e layout do mapa:

- abaixo de 0,499: desenvolvimento humano (muito baixo) - vermelho;

$-0,500$ até 0,599: desenvolvimento humano (baixo) - laranja;

$-0,600$ até 0,699: desenvolvimento humano (médio) - amarelo;

$-0,700$ até 0,799: desenvolvimento humano (alto) - verde;

- acima de 0,800: desenvolvimento humano (muito alto) - azul.

Para o estabelecimento de comparação dos dados desta pesquisa com a de outros autores relativo aos resultados do IDHM de 2000, as classes foram renomeadas conforme a nova classificação do IDHM de 2010 (ATLAS BRASIL, 2013).

A distribuição espacial foi realizada segundo as técnicas da cartografia temática, operacionalizada por meio do software ArcGis ${ }^{\circledR}$ (ENVIRONMENTAL..., 2007). Na classe de médio desenvolvimento humano distribuiu-se a cor amarela em quatro tonalidades para diferenciar os municípios no mapa.

\section{RESULTADOS E DISCUSSÃO}

Conforme as análises, os municípios fundados no século 18 apresentaram os meIhores resultados nos indicadores de renda $(\mathrm{m})$ (alto desenvolvimento humano) e educação $(\mathrm{m})$ (médio desenvolvimento). Os municípios emancipados no período de 1913 a 1963 exibiram o indicador de longevidade $(\mathrm{m})$ (muito alto) superior aos demais períodos analisados. Os emancipados no período compreendido entre 1811 a 1861 sustentaram os valores mais baixos nos indicadores de renda $(\mathrm{m})$ (médio), longevidade $(\mathrm{m})$ (alto) e educação (m) (baixo) (Figura 2).

Figura 2 - IDHM (m) dos municípios distribuídos nos períodos de fundação/emancipação

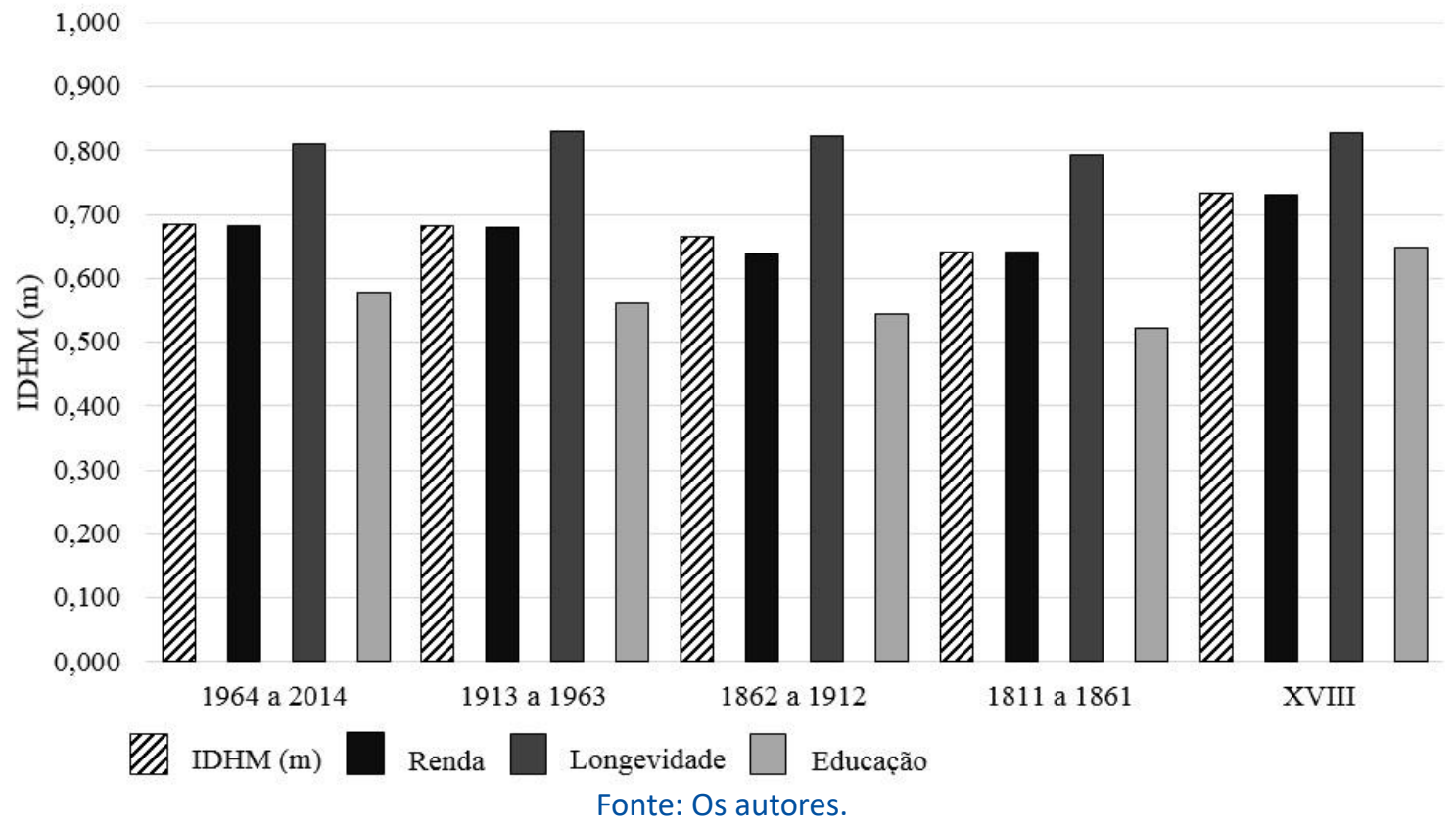


O IDHM (m) nos períodos de fundação e/ou emancipação, representado por Cáceres/MT, Cuiabá/MT e Corumbá/MS (século 18), foi de alto desenvolvimento humano, e os demais municípios caracterizaram-se em médio desenvolvimento, com resultados mais aquém os municípios emancipados entre os anos de 1811 a 1861 (Miranda/MS e Poconé/MT) (Figura 3).

Figura 3 - Espacialização do IDHM (m) dos municípios no bioma Pantanal nos períodos de fundação e/ou emancipação

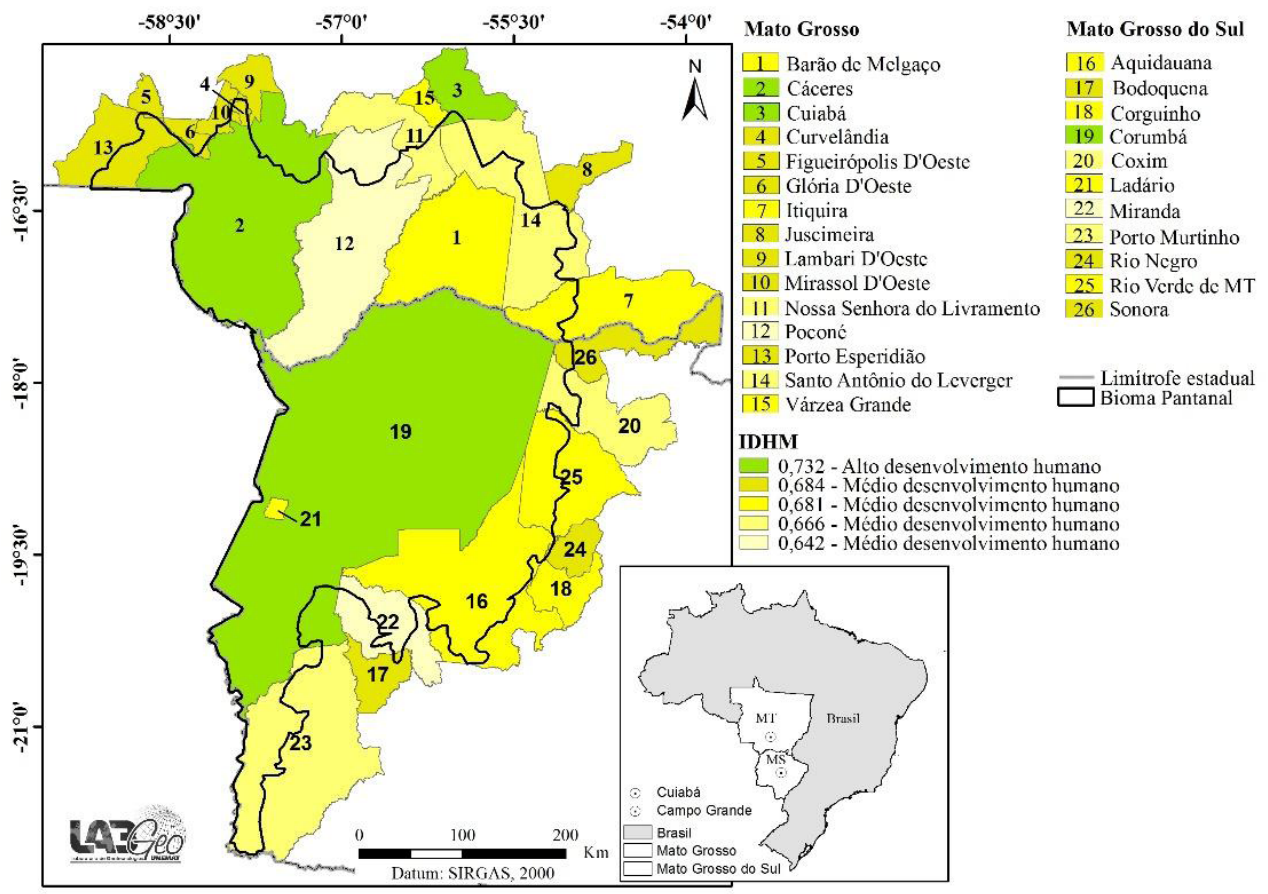

Fonte: UNIVERSIDADE..., 2017.

A partir do levantamento observou-se que o melhor índice do IDHM dos municípios analisados foi da capital de Mato Grosso, Cuiabá, posicionando-se em alto desenvolvimento humano. Barão de Melgaço, também no Estado de Mato Grosso, registrou o menor índice (médio desenvolvimento).

As possíveis causas para esses e outros resultados do IDHM referem-se ao perfil do administrador público (gestão), às políticas executadas no município, à infraestrutura, à localização geográfica e às riquezas naturais do território, entre outros fatores que, decididamente, influenciam no desenvolvimento humano e são pré-requisitos discutidos por Buarque (2006) e Saquet (2007).

Longevidade foi o indicador com melhor desempenho no IDHM alavancado pelo município de Rio Negro/MS (muito alto) e, na contramão, Bodoquena/MS, mesmo assim considerado como alto desenvolvimento.

Renda foi o segundo melhor indicador e Cuiabá/MT o representou (muito alto desenvolvimento). Na oposição, o município de Barão de Melgaço/MT situou-se em baixo desenvolvimento.

$O$ indicador de educação, com resultados inferiores aos demais, teve como melhor desempenho o município de Cuiabá/MT, em alto desenvolvimento humano. Barão de Melgaço/MT, novamente, apresentou os menores índices, encontrando-se na faixa de muito baixo. 
Barão de Melgaço/MT, Bodoquena/MS, Cuiabá/MT e Rio Negro/MS foram os quatro municípios de destaque, positivos e negativos, diante das constatações. Para entender melhor esses dados desagregou-se os indicadores do IDHM (Tabela 1).

Tabela 1 - Indicadores desagregados do IDHM dos municípios de destaque

\begin{tabular}{lcrrr}
\hline Indicadores & $\begin{array}{c}\text { Barão de } \\
\text { Melgaço/MT }\end{array}$ & Bodoquena/MS C & uiabá/MT & $\begin{array}{r}\text { Rio } \\
\text { Negro/MS }\end{array}$ \\
\hline População & 7.5917 & .9855 & 51.098 & 5.036 \\
Mortalidade infantil & 16,4 & 8,2 & 13,2 & 34,5 \\
Esperança de vida & 74,2 & 71,57 & 5,0 & 77,2 \\
Renda per capita & 330,005 & 00,00 & $1.160,00$ & 631,00 \\
Salário médio mensal & 1,6 & 2,6 & 3,6 & 1,8 \\
Gini & 0,50 & 0,50 & 0,59 & 0,54 \\
Ideb $^{2}$ & 3,9 & 4,3 & 4,1 & 3,2 \\
\hline
\end{tabular}

* Resultados grifados em verde expressam o melhor resultado e em amarelo o pior. ${ }^{1}$ Com base no salário mínimo de 2010 (R\$ 510,00). ${ }^{2}$ Índice de Desenvolvimento da Educação Básica (Ideb).

Fonte: INSTITUTO...,2010; ATLAS BRASIL, 2013.

Para o indicador de longevidade, o município de Rio Negro/MS apresentou o meIhor resultado em relação à esperança de vida ao nascer e o pior no indicador de mortalidade de crianças com menos de um ano de idade, situação reversa no município de Bodoquena/MS.

No indicador de renda, Cuiabá/MT sobressaiu-se na renda per capita e no salário médio mensal e, na contramão, Barão de Melgaço/MT. As posições se inverteram quando se analisou a concentração de renda (Gini), com destaque positivo, também, para Bodoquena/MS.

No Ideb, índice que avalia o desenvolvimento da educação básica, não se encontrou uma relação clara com o indicador de educação, pois o melhor resultado constatou-se no município de Bodoquena e o pior em Rio Negro, ambos no Mato Grosso do Sul.

Em continuidade, Cuiabá foi o primeiro e mais antigo município fundado em Mato Grosso e sustenta o melhor IDHM (alto), e os indicadores de renda (muito alto), longevidade (muito alto - compartilhado com Corumbá/MS) e educação (alto) no período de análise. Cáceres/MT e Corumbá/MS foram fundadas no mesmo ano, entretanto o primeiro registrou os menores indicadores de renda (médio) e longevidade (muito alto), e o segundo os menores valores no IDHM (alto) e em educação (baixo) (Tabela 2).

Tabela 2 - Indicadores do IDHM para os municípios fundados no século 18

\begin{tabular}{lrrrrr}
\hline Municípios/estado & Fundação & IDHM & Renda & Longevidade & Educação \\
\hline Cáceres/MT & 1778 & 0,708 & 0,691 & 0,813 & 0,633 \\
Corumbá/MS & 1778 & 0,700 & 0,701 & 0,834 & 0,586 \\
Cuiabá/MT & 1719 & 0,785 & 0,800 & 0,834 & 0,726 \\
\hline IDHM (m) & & 0,732 & 0,731 & 0,827 & 0,648
\end{tabular}

* Resultados grifados em verde expressam o melhor resultado e em amarelo o pior. Fonte: Adaptado da ASSOCIAÇÃO..., 2017; ATLAS BRASIL, 2013; SECRETARIA..., 2017. 
Azevedo (2013) conferiu o IDHM dos municípios criados no primeiro período (século 18) no Rio Grande do Sul e no Piauí e constatou que o desenvolvimento humano de uma região à outra foi adverso em favor do primeiro. Nesse sentido, Fleury (2003) discutiu que o processo de emancipação não representa, necessariamente, alterações significativas nas condições sociais e econômicas.

Entre 1811 a 1861, dois municípios foram emancipados, e o melhor resultado do IDHM (médio), renda (médio), longevidade (muito alto) e educação (baixo) encontrou-se em Poconé/MT, distinto do verificado em Miranda/MS, que foi médio, médio, alto e baixo desenvolvimento, respectivamente (Tabela 3).

O melhor IDHM para aqueles municípios emancipados entre 1862 a 1912 (Tabela 4) foi encontrado em Coxim/MS (alto), para os indicadores de renda (alto), longevidade (muito alto) e educação (baixo). Nossa Senhora do Livramento/MT acumulou o IDHM e renda como médio desenvolvimento. Santo Antônio do Leverger/MT apresentou o menor valor em longevidade (muito alto), mesmo assim todos os municípios estão situados em alto desenvolvimento. $O$ baixo desenvolvimento no indicador de educação foi representado por Porto Murtinho/MS.

Tabela 3 - Indicadores do IDHM para os municípios emancipados entre os anos de 1811 e 1861

\begin{tabular}{lrrrrr}
\hline Municípios/Estado & Emancipação & IDHM & Renda & Longevidade & Educação \\
\hline Miranda/MS & 1857 & 0,632 & 0,638 & 0,782 & 0,507 \\
Poconé/MT & 1831 & 0,652 & 0,643 & 0,806 & 0,534 \\
\hline IDHM (m) & & 0,642 & 0,641 & 0,794 & 0,521
\end{tabular}

* Resultados grifados em verde expressam o melhor resultado e em amarelo o pior.

Fonte: Adaptado da ASSOCIAÇÃO..., 2017; ATLAS BRASIL, 2013; SECRETARIA..., 2017.

Tabela 4 - Indicadores do IDHM para os municípios emancipados entre os anos de 1862 e 1912

\begin{tabular}{lrrrrr}
\hline Municípios/Estado & Emancipação & IDHM & Renda & Longevidade & Educação \\
\hline Porto Murtinho/MS & 1911 & 0,666 & 0,677 & 0,830 & 0,526 \\
Coxim/MS & 1898 & 0,703 & 0,719 & 0,836 & 0,579 \\
Santo Antônio do Leverger/MT & 1890 & 0,656 & 0,651 & 0,806 & 0,539 \\
Nossa Senhora do & 1883 & 0,638 & 0,600 & 0,820 & 0,529 \\
Livramento/MT & & & & & \\
\hline IDHM (m) & & 0,666 & 0,662 & 0,823 & 0,543 \\
\hline
\end{tabular}

* Resultados grifados em verde expressam o melhor resultado e em amarelo o pior.

Fonte: Adaptado da ASSOCIAÇÃO..., 2017; ATLAS BRASIL, 2013; SECRETARIA..., 2017.

Matei e Filippi (2013) estudaram o município de Santa Vitória do Palmar/RS, emancipada durante o terceiro período, e encontraram o IDHM de 2000 superior ao registrado pelos munícipios contidos no bioma Pantanal, bem como os melhores indicadores para educação, renda e longevidade, em ordem de classificação, distinto da pesquisa que foi longevidade, renda e educação.

No período de 1913 a 1963 o município de Várzea Grande/MT registrou o melhor desempenho no IDHM (alto) e nos indicadores de renda (alto) e educação (médio), situação oposta ao encontrado em Barão de Melgaço/MT, que acumulou o menor valor 
do IDHM (médio), seguido pelo indicador de renda (baixo) e educação (muito baixo). No indicador de longevidade todos os municípios situaram-se com alto desenvolvimento humano, entretanto Itiquira/MS apresentou o menor valor e Rio Verde de Mato Grosso/MS o maior (Tabela 5).

Tabela 5 - Indicadores do IDHM para os municípios emancipados entre os anos de 1913 e 1963

\begin{tabular}{lrrrrr}
\hline Municípios/Estado & Emancipação & IDHM & Renda & Longevidade & Educação \\
\hline Barão de Melgaço/MT & 1953 & 0,600 & 0,598 & 0,820 & 0,440 \\
Itiquira/MT & 1953 & 0,693 & 0,694 & 0,812 & 0,591 \\
Corguinho/MS & 1953 & 0,671 & 0,684 & 0,830 & 0,531 \\
Ladário/MS & 1953 & 0,704 & 0,687 & 0,822 & 0,618 \\
Rio Verde de Mato Grosso/MS & 1953 & 0,673 & 0,686 & 0,852 & 0,521 \\
Várzea Grande/MT & 1948 & 0,734 & 0,711 & 0,842 & 0,661 \\
Aquidauana/MS & 1918 & 0,688 & 0,690 & 0,840 & 0,562 \\
\hline IDHM (m) & & 0,681 & 0,679 & 0,831 & 0,561 \\
\hline
\end{tabular}

* Resultados grifados em verde expressam o melhor resultado e em amarelo o pior. Fonte: Adaptado da ASSOCIAÇÃO..., 2017; ATLAS BRASIL, 2013; SECRETARIA..., 2017.

Assunção, Melo e Soares (2007) verificaram o IDHM de 2000 dos pequenos municípios da microrregião de Catalão/GO, cuja grande parte foi emancipada entre os anos de 1931 e 1953, e constataram que o índice variou de alto a muito alto. Boccaletto (2007) destacou o IDHM de 2000 do município de Vinhedo/SP, emancipado no segundo período, como muito alto.

Assunção, Melo e Soares (2007) apontaram os melhores resultados do IDHM em educação, longevidade e renda da microrregião de Catalão/GO, diferente ao encontrado por esta pesquisa, que foi longevidade, renda e educação. O melhor desempenho do indicador longevidade os autores atribuíram ao fato do elevado número de idosos nas pequenas cidades.

A distribuição dos municípios por período possibilitou compreender que dentre os emancipados de 1964 a 2014 o de menor IDHM foi Lambari D’Oeste/MT (médio), acumulando também o indicador de educação (muito baixo). Juscimeira/MT registrou o melhor IDHM (alto) e o indicador de educação (médio). No indicador de renda, Curvelândia/MT (médio) assumiu o posto contrário ao de Mirassol D’Oeste/MT (alto), assim como Bodoquena/MS (alto) a Rio Negro/MS (muito alto) no indicador de longevidade (Tabela 6).

Matias e Galindo (2011) e Camargo (2013) averiguaram o IDHM (de 2000) de Paulínia/SP e Flores da Cunha/RS, respectivamente, emancipados nos últimos 50 anos, e constataram que esses se apresentaram como muito alto, similar ao encontrado por Azevedo (2011) para os municípios do Estado do Piauí e Rio Grande do Sul, no qual os mais novos possuem IDHM melhor do que aqueles emancipados antes de 1980. 
Tabela 6 - Indicadores do IDHM para os municípios emancipados entre os anos de 1964 e 2014

\begin{tabular}{lrrrrr}
\hline Municípios/Estado & Emancipação & IDHM & Renda & Longevidade & Educação \\
\hline Curvelândia/MT & 1998 & 0,690 & 0,658 & 0,811 & 0,615 \\
Glória D'Oeste/MT & 1991 & 0,710 & 0,684 & 0,823 & 0,636 \\
Lambari D'Oeste/MT & 1991 & 0,627 & 0,670 & 0,787 & 0,467 \\
Sonora/MS & 1988 & 0,681 & 0,706 & 0,803 & 0,557 \\
Figueirópolis D’Oeste/MT & 1986 & 0,679 & 0,665 & 0,786 & 0,599 \\
Porto Esperidião/MT & 1986 & 0,652 & 0,666 & 0,795 & 0,523 \\
Bodoquena/MS & 1980 & 0,666 & 0,665 & 0,776 & 0,573 \\
Juscimeira/MT & 1979 & 0,714 & 0,678 & 0,842 & 0,637 \\
Mirassol D'Oeste/MT & 1976 & 0,704 & 0,723 & 0,816 & 0,592 \\
Rio Negro/MS & 1964 & 0,709 & 0,702 & 0,869 & 0,585 \\
\hline IDHM (m) & & 0,684 & 0,682 & 0,811 & 0,578
\end{tabular}

Fonte: Adaptado da ASSOCIAÇÃO..., 2017; ATLAS BRASIL, 2013; SECRETARIA..., 2017.

Em última análise, os municípios que compõem o Estado de Mato Grosso do Sul apresentaram os melhores índices do IDHM, renda (ambos em alto desenvolvimento humano) e longevidade (muito alto) e o Brasil no indicador de educação (médio). Situação contrária foi observada com os 15 municípios de Mato Grosso situados no bioma que representaram o indicador de renda $(\mathrm{m})$ (médio) e longevidade $(\mathrm{m})$ (muito alto) abaixo dos demais, assim como para os 11 municípios pantaneiros do Mato Grosso do Sul no índice do IDHM (m) (médio) e educação (m) (baixo) (Tabela 7).

Desses dados alguns fatores merecem observações à parte: os índices de desenvolvimento humano, tanto dos municípios inseridos no bioma mato-grossense quanto os no bioma sul-mato-grossense, foram inferiores ao comparar com os Estados (MT e MS, nesta ordem) e com o Brasil; com exceção da longevidade, os outros indicadores dos municípios pantaneiros situaram-se bem abaixo dos Estados e do Brasil.

Tabela 7 - IDHM e indicadores das unidades territoriais do bioma Pantanal, Estados e do Brasil

\begin{tabular}{lrrrr}
\hline Região & IDHM & Renda & Longevidade & Educação \\
\hline Municípios do bioma em Mato Grosso' $^{1}$ & 0,684 & 0,675 & 0,814 & 0,581 \\
Municípios do bioma em Mato Grosso do Sul $^{1}$ & 0,681 & 0,687 & 0,825 & 0,559 \\
Municípios do bioma pantaneiro' $^{1}$ & 0,683 & 0,680 & 0,819 & 0,572 \\
Mato Grosso & 0,725 & 0,732 & 0,821 & 0,635 \\
Mato Grosso do Sul & 0,729 & 0,740 & 0,833 & 0,629 \\
Brasil & 0,727 & 0,739 & 0,816 & 0,637 \\
\hline
\end{tabular}

* Resultados grifados em verde expressam o melhor resultado e em amarelo o pior. ${ }^{1}$ IDHM (m), renda (m), longevidade $(m)$ e educação $(m)$.

Fonte: Adaptado do ATLAS BRASIL, 2013.

Destarte, nos últimos 66 anos (1948 a 2014) foram emancipados 16 municípios dos 26 presentes no bioma Pantanal, fator muito provavelmente relacionado aos fluxos migratórios e no avanço da fronteira agropecuária no Brasil, cujas ações referem-se 
às do governo de Getúlio Vargas (Marcha para o Oeste) e dos programas surgidos durante os governos militares na década de 60 do século 20 (Polocentro e Polonoroeste) (CUNHA, 2003; RAMMINGER; GRASEL; ZAVALA, 2009).

Essas medidas influenciaram na ocupação territorial do grande Estado de Mato Grosso que, em 1977, teve parte de seu território desmembrado para a criação do Estado de Mato Grosso do Sul (CHATE et al., 2009) resultando, na concepção de Santos (2008), em um novo processo de urbanização dos distritos, industrialização e execução e/ou ampliação da infraestrutura das cidades.

Emancipar, entretanto, os distritos ou as unidades territoriais sem a devida organização, a citar a estrutura física, social, econômica, política e administrativa, contextos presentes no Plano Diretor (VILLAÇA, 1999) e ressaltado por Batista Filho e Borba (2013), que ainda acrescenta a temática ambiental, possivelmente comprometerá o desenvolvimento humano, limitando as possibilidades de escolhas da população, ou seja, das condições e liberdades para fazer e ser na sua vida (SEN, 2010).

\section{CONCLUSÕES}

Baseando-se na argumentação teórica e nos resultados encontrados defende-se que o desenvolvimento humano não pode ser medido apenas por questões econômicas, mas, sim, em uma interação de diversos pressupostos: humanos, sociais, físicos, econômicos, administrativos e ambientais.

A ausência de um ou mais pressupostos muito provavelmente impactará na capacidade ou liberdade dos demais, ou seja, na qualidade de vida dos moradores (saudável, participativa e livre) e na própria sustentabilidade socioestrutural da criação de novos municípios.

A emancipação que não observa a sustentabilidade socioestrutural pode resultar, dessa forma, na criação de municípios sem as características básicas de manutenção e de suporte ao desenvolvimento humano, o que implicará na qualidade de vida de seus habitantes, a exemplo dos municípios de Barão de Melgaço/MT e Bodoquena/MS, que apresentaram os valores mais baixos nos indicadores de desenvolvimento humano.

A partir das análises expostas concluiu-se que o desenvolvimento humano da fundação e/ou emancipação dos municípios não se associou diretamente com os períodos de sua criação, isso quer dizer que os mais antigos, de maneira geral, não se sobressaíram aos mais novos, e vice-versa.

Ademais, questões como o perfil da gestão e administração pública, políticas públicas, localização geográfica, riquezas naturais, infraestrutura, etc., podem contribuir significativamente com os resultados do IDHM (tanto positiva quanto negativamente), como foram os casos de Cuiabá e Barão de Melgaço.

\section{REFERÊNCIAS}

ABDON, M. M. et al. Desmatamento no bioma pantanal até o ano 2002: relações com a fitofisionomia e limites municipais. Revista Brasileira de Cartografia, Rio de Janeiro, v. 1, n. 59, p. 17-24, abr. 2007.

ABRUCIO, F. L.; FRANZESE, C. Federalismo e políticas públicas: o impacto das relações intergovernamentais no Brasil. In: ARAÚJO, M. F. I.; BEIRA, L. (Org.). Tópicos de economia paulista para gestores públicos. São Paulo: Fundap, 2007. p. 13-31.

ALBA, R. S. Espaço urbano: os agentes da produção em Chapecó. Chapecó: Argos, 2002.

ASSOCIAÇÃO MATO-GROSSENSE DOS MUNICÍPIOS (AMM). Emancipação. Cuiabá: AMM, 2017. Disponível em: <http://www.amm.org.br/amm/emancipacao>. Acesso em: 2 fev. 2017. 
ANGEOLETTO, F. et al. Tipología socio-ambiental de las ciudades medias de Brasil: aportes para un desarrollo urbano sostenible. Urbe, Curitiba, v. 8, n. 2, p. 272-87, maio/ago. 2016.

ASSUNÇÃO, M. C. F.; MELO, N. A.; SOARES, B. R. Caracterização sócio-econômica e espacial das pequenas cidades da microrregião de Catalão (GO). Horizonte Científico, Uberlândia, v. 1, n. 1, p. 1-32, jan./dez. 2007.

ATLAS BRASIL. Atlas do Desenvolvimento Humano no Brasil 2013. Brasília, DF: PNUD; FJP; IPEA, 2013. Disponível em: <http://www.atlasbrasil.org.br/2013/>. Acesso em: 2 fev. 2017.

AZEVEDO, D. A. O contexto espacial como um balizador de fenômenos sociais: o caso das emancipações municipais no Rio Grande do Sul e Piauí. Boletim Gaúcho de Geografia, Porto Alegre, v. 40, n. 1, p. 53-71, maio 2013.

Isonomia e território no Brasil. Revista Geográfica de América Central, Heredia, jul./dez. 2011. (número especial).

BATISTA FILHO, M.; BORBA, J. Desenvolvimento humano e saúde no Brasil. Revista Brasileira de Saúde Materno Infantil, Recife, v. 13, n. 3, p. 1-2, jul./set. 2013.

BOCCALETTO, E. M. A. Características socioeconômicas do município de Vinhedo/SP. In: BOCCALETTO, E. M. A.; VILARTA, R. (Org.). Diagnóstico da alimentação saudável e atividade física em escolas municipais de Vinhedo/SP. Campinas: Ipes Editorial, 2007. p. 17-24.

BRASIL. Ministério do Meio Ambiente. Pantanal. Brasília, DF: MMA, 2017. Disponível em: <http://www. mma.gov.br/biomas/pantanal>. Acesso em: 28 jan. 2017.

BUARQUE, S. C. Construindo o desenvolvimento local sustentável: metodologia de planejamento. 3. ed. Rio de Janeiro: Garamond, 2006.

CAMARGO, R. Proposta de zoneamento ambiental para o município de Flores da Cunha-RS. 2013. Monografia (Graduação) - Faculdade de Geografia da Universidade Regional do Noroeste do Estado do Rio Grande do Sul, Unijuí, ljuí, 2013.

CHATE, S. C. et al. Epidemiologic situation of bovine brucellosis in the state of Mato Grosso, Brazil. Arquivo Brasileiro de Medicina Veterinária e Zootecnia, Belo Horizonte, v. 61, n. 1, p. 56-65, nov. 2009.

CIGOLINI, A. A. Ocupação do território e criação de municípios no período republicano brasileiro. Biblio 3W, Barcelona, v. 19, n. 1.101, p. 1-15, dez. 2014.

CUNHA, J. M. P. Redistribuição espacial da população: tendências e trajetória. São Paulo em Perspectiva, São Paulo, v. 17, n. 3-4, p. 218-33, jul./dez. 2003.

EMPRESA BRASILEIRA DE PESQUISA AGROPECUÁRIA (Embrapa). O Estado do Mato Grosso. Campinas: Embrapa, 2017. Disponível em: <http://www.qmdmt.cnpm.embrapa.br/711.htm>. Acesso em: 26 jan. 2017.

ENVIRONMENTAL SYSTEMS RESEARCH INSTITUTE (Esri). ArcGIS Desktop: release 9.2. Redlands, CA: Esri, 2007.

FERNANDES, A. S. A; ARAÚJO, S. M. V. G. A criação de municípios e a formalização de regiões metropolitanas: os desafios da coordenação federativa. Urbe, Curitiba, v. 7, n. 3, p. 295-309, set./dez. 2015.

FLEURY, S. F. Emancipação de municípios: um exame de indicadores. Revista do Legislativo, Belo Horizonte, n. 37, p. 58-73, jul./dez. 2003.

HAQ, M. ul. Reflections on human development. New York: Oxford University Press, 1995.

INSTITUTO BRASILEIRO DE GEOGRAFIA E ESTATÍSTICA (IBGE). 2010, 2014.

MACHADO, J. G. R.; PAMPLONA, J. B. A ONU e o desenvolvimento econômico: uma interpretação das bases teóricas da atuação do PNUD. Economia e Sociedade, Campinas, v. 17, n. 1, p. 53-84, abr. 2008.

MATEI, A. P.; FILIPPI, E. E. O bioma pampa e o desenvolvimento socioeconômico em Santa Vitória do Palmar. Ensaios FEE, Porto Alegre, v. 34, p. 739-64. 2013. (número especial).

MATIAS, L. F.; GALINDO, C. A. Geoprocessamento aplicado à análise das transformações no uso da terra no município de Paulínia-São Paulo, Brasil. Engenharia Civil, Guimarães, n. 40, p. 17-26, maio 2011.

MORENO, G. Agricultura: transformações e tendências. In: MORENO, G.; HIGA, T. C. (Org.). Geografia de Mato Grosso: território, sociedade, ambiente. Cuiabá: Entrelinhas, 2005. p. 140-71.

PITTERI, S. Desenvolvimento econômico sustentável dos pequenos municípios paulistas. Revista de Desenvolvimento Econômico, Salvador, v. 8, n. 11, p. 46-56, jan. 2005.

RAMMINGER, R.; GRASEL, D.; ZAVALA, A. A. Z. Agricultura e indicadores de renda no Estado de Mato Grosso (1980-2005). Informe Gepec, Cascavel, v. 12, n. 2, p. 56-72, jul./dez. 2008.

SANTOS, M. A urbanização brasileira. 5. ed. São Paulo: Edusp, 2008.

SAQUET, M. A. Abordagem e concepções sobre território. São Paulo: Expressão Popular, 2007. 
SECRETARIA DE ESTADO DE MEIO AMBIENTE, DO PLANEJAMENTO, DA CIÊNCIA E TECNOLOGIA DE MATO GROSSO DO SUL (Semac-Estado). Cuiabá: Semac. Disponível em: <http://www.semac.ms.gov.br/>. Acesso em: 2 fev. 2017.

SEN, A. Desenvolvimento como liberdade. São Paulo: Companhia das Letras, 2010.

TORRES, H. G.; FERREIRA, M. P.; DINI, N. P. Indicadores sociais: por que construir novos indicadores como o IPRS. São Paulo em Perspectiva, São Paulo, v. 17, n. 3-4, p. 80-90, jul./dez. 2003.

UNIVERSIDADE DO ESTADO DE MATO GROSSO (Unemat). Laboratório de Geotecnologias (Labgeo/Unemat). 2017.

UNITED NATIONS DEVELOPMENT PROGRAMME (UNDP). Human Development Report 1990. New York: Oxford University Press, 1990.

Human Development Report 1992. New York: Oxford University Press, 1992.

Human Development Report 2001. New York: Oxford University Press, 2001.

Human Development Report 2014. New York: Oxford University Press, 2014.

VEIGA, J. E. Cidades imaginárias: o Brasil é menos urbano do que se calcula. Campinas: Autores Associados, 2003.

VILLAÇA, F. Dilemas do plano diretor. In: CEPAM. O município no século XXI: cenários e perspectivas. São Paulo: Cepam, 1999. p. 237-47. 